\title{
Acknowledgement of Reviewers for 2019
}

Please join us in thanking all those scientists and experts in the various fields represented in Mathematical Geosciences for devoting time and effort to reviewing the papers that we have sent them. The Editor-in-Chief, the IAMG and Publisher acknowledge the colleagues listed below for their excellent reviews of papers for which final decisions were made during the period 1 January 2019 to 31 December 2019.

Petter Abrahamsen

Arash Adib

Christian Agrell

Luc Alberts

Teresa Albuquerque

Denis Allard

Tatiyana Apanasovich

Daisy Arroyo

Peter Atkinson

Per Åge Avseth

Leonardo Azevedo

Susana Barbosa

Daniel Barker

Anne Bartetzko

Ludmila Belyakova

Giorgos Benndorf

Brian Berkowitz

Mara Sabina Bernardi

Laurent Bertino

Lin Bi

Katharina Billups

Ettore Biondi
Madalyn Blondes

Neven Bočić

Gilles Bourgault

Antonella Buccianti

Dieu Tien Bui

Nikita Bukhanov

Ilaria Butera

Anzhou Cao

Zi-Jun Cao

James Carr

Guillaume Caumon

Bishwajit Chakraborty

Snehamoy Chatterjee

Fa Chen

Yangkang Chen

Yongliang Chen

David Cohen

Alessandro Comunian

Patrick Connolly

Joao Felipe Costa

Massimiliano Cremonesi

Noel Cressie 
Franck Curriero

Camilla Zacche da Silva

Guillaume Damiand

Sandra De Iaco

Eric de Kemp

Jared Deutsch

Michele Dragoni

Fabrice Dupros

Carlos Duque

Fabien Durand

Bergur Einarsson

Jean Elkoury

Ahmed Elsheikh

Xavier Emery

Gabriel Fabien-Ouellet

Fangxin Fang

James Farquharson

Eric Fetzer

Maria Dolores Fidelibus

Francky Fouejio

Jianlin Fu

Kerry Gallagher

Robert Garrett

Yong Ge

Hadi Ghofrani

Richard Gibson

Vasileios-Marios Gkortsas

Erwan Gloaguen

J. Jaime Gómez-Hernández

Álvaro González

Ryan Goodfellow

Pierre Goovaerts

Eric Grunsky

James Gunning

Jingcheng Han

Paul Harris

Denis Heliot

Y. Huang

Raphael Huser

Attila Imre

Joaquín Irazábal González

Daniela Jacob

Behnam Jafarpour

Saman Javadis

Tómas Jóhannesson

Martin Jullum
Mikhail Kanevski

Gordon Kaufman

Boris Kaus

Nina Kirchner

Harald Klammler

Amir Kolahi-Azar

Odd Kolbjornsen

Alexander Kolovos

Karel Kovarik

Devesh Kumar

Annika Lang

Christian Lantuejoul

Pauline Le Bouteiller

Bernard Legras

Ting Lei

Chunhui Li

Fangyu Li

Liangping $\mathrm{Li}$

S. Y. Li

Xue Li

X. San Liang

Niklas Linde

Chris Lloyd

Xianqing Lv

Steve Lyster

Yuan Ma

Nasser Madani

Vahid Mahboub

Denis Marcotte

Ryan Martin

Barbara Martinucci

Sean McKenna

Jennifer McKinley

Giacomo Medici

Alessandra Menafoglio

Baehyun Min

Ilnur Minniakhmetov

Hossein Mojaddadi Rizeei

Matty Mookerjee

Ute Mueller

Tapan Mukerji

Donald Myers

Benoit Noyelles

Ricardo Olea

Julian Ortiz

Muhammed Öztürk 
Hari Pandalai

Eulogio Pardo Iguzquiza

Vera Pawlowsky-Glahn

Jon Pelletier

Lorenzo Perozzi

Geoffrey Phelps

Dimitrii Posvyanskiy

Andrés Prieto

Michael Pyrcz

Andres Quiros

Philippe Renard

Atish Roy

Elena Savelieva

Celine Scheidt

Ernst Schetselaar

John Schuenemeyer

Chan Shing

Donald Singer

Thomas Slater

Amilcar Soares

Louise Sørensen

Laurent Souche

Mohan Srivastava

Sebastien Strebelle

Keli Sun

Satomi Suzuki
Reza Taherdangkoo

Pejman Tahmasebi

Hassan Talebi

Yunwei Tang

Michael Thiel

Raimon Tolosana-Delgado

Peter Varkonyi

Surendra Verma

Bin Wang

Jeen-Hwa Wang

Wenlei Wang

Xiao Wang

Mauro Werder

Chicheng Xu

Chuntang Xu

Guangping $\mathrm{Xu}$

Xiuwei Yang

Lingqing Yao

M. Yousefi

Yevgeniy Zagayevskiy

Ayham Zaitouny

Jingxiong Zhang

Junfeng Zhang

Tuanfeng Zhang

Renguang Zuo 